\title{
Un enfoque para la optimización de pagos móviles para el sistema de transporte utilizando (NFC) a través de Cloud Computing
}

\section{(An Approach to the Optimization of Mobile Payments for the Transport System using (NFC) through Cloud Computing)}

\author{
Estevan R Gómez Torres, Nelson Herrera-Herrera ${ }^{1}$, Magi Paul Díaz ${ }^{2}$
}

\begin{abstract}
Resumen:
Cada vez el uso de dispositivos móviles para realizar pagos se ha ido incrementando. Día a día son más las organizaciones que adoptan sistemas que incluyen algún sistema de pagos móvil, por ello es necesario contar con sistemas rápidos y ágiles que permitan garantizar la seguridad y confiabilidad, tanto para el operador como para el mismo usuario, a fin de que los usuarios obtengan un servicio de calidad basado en las tecnologías móviles. En éste trabajo se realiza un análisis de la tecnología NFC tomando en cuenta una propuesta de desarrollo de un sistema de medios de pago electrónicos, aplicable al Metro de Quito, para asegurar el tiempo de respuesta y la seguridad transaccional, se adopta la solución de sistemas en la Nube.
\end{abstract}

Palabras clave: NFC, Aplicaciones Móviles, Computación en la Nube, Soluciones de Pagos en Transporte

\begin{abstract}
:
The use of mobile devices has gradually increased. Every day, the number of organizations adopting systems which include some type of mobile payments is becoming bigger. Because of this reason, it is mandatory to have agile and quick systems to guarantee security and reliability, not only for the user but also for the operator. In that way, users will get a high-quality service based on mobile technologies. An analysis of NFC Technology has been made in this paper taking into consideration a proposal of a system development including mobile payments, which could be used in the transportation system of Metro of Quito. To assure the response time and transactional security, the use of cloud computing is recommended.
\end{abstract}

Keywords: NFC, Mobile applications, Cloud Computing, Transporting payment Solutions

\section{Introducción}

Las comunicaciones de campo cercano (NFC) muy populares por su versatilidad y aplicabilidad, surgen como alternativas para enviar y recibir datos, con la presencia de un emisor y un receptor, como características principales se tiene: está diseñada para la transmisión instantánea de pequeñas cantidades de información como es el caso y su forma de operar determina que sea de muy corto alcance, pues trabaja a 13,56 MHZ con una tasa de transferencia de información puede alcanzar los $424 \mathrm{Kbps}$ y lo hace usando una tecnología inalámbrica para transmisión de datos. Es

\footnotetext{
${ }^{1}$ Universidad Tecnológica Equinoccial, Quito - Ecuador ( \{estevan.gomez, Nelson.herrera\} @ute.edu.ec )

${ }^{2}$ Universidad de las Fuerzas Armadas (Espe), Quito- Ecuador( mpdiaz@espe.edu.ec)
} 
así que NFC tiene en éstos momentos mucho soporte debido a lo cual, se encuentra presente en la gran mayoría de teléfonos inteligentes y otros dispositivos móviles. Además, se puede considerar como un estándar creado para la comunicación sin cables de corto alcance para la realización de pagos a través de dispositivos móviles mayoritariamente. El resultado es un incremento sostenido de las aplicaciones que utilizan NFC, en diversos campos como: Movilidad Inteligente, Producción Inteligente, Marketing Inteligente, Control de Pagos y Accesos, Viviendas controladas y conectadas, lo cual le da un rango de cobertura y aplicación muy amplio a las tecnologías NFC (Romero, 2017), como se observa en la Figura 1.

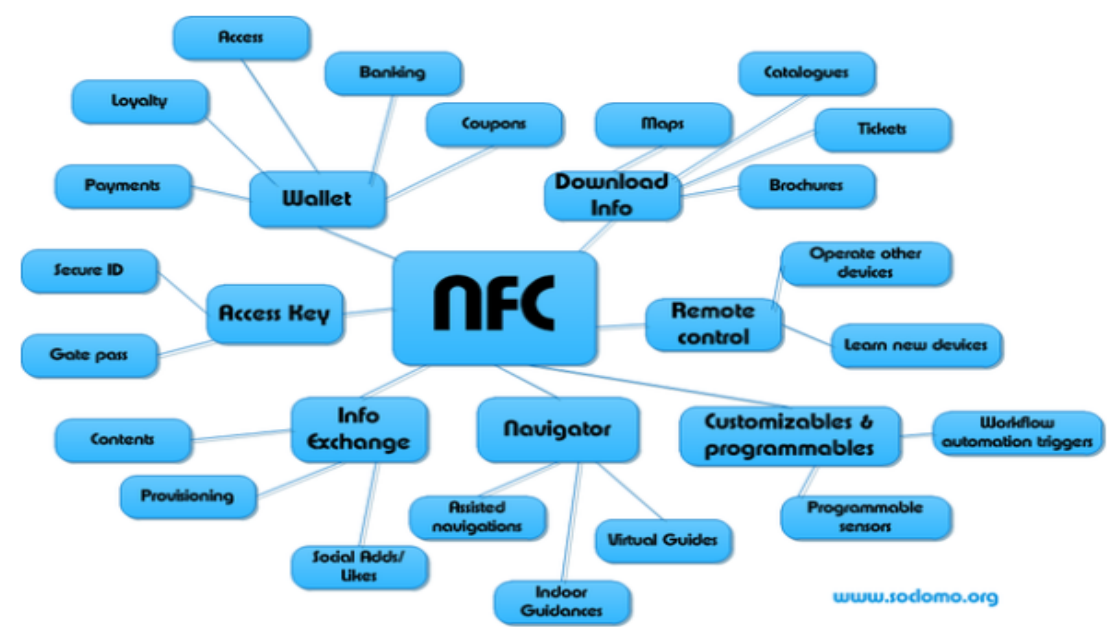

Figura 1: APLICACIONES NFC

Un cambio drástico en la forma de interactuar con los objetos, ha sido introducido por el Internet de las Cosas (IoT), es así como se han creado múltiples dispositivos que aprovechan ésta tecnología NFC y que pueden perfectamente ser utilizados en el hogar, en el trabajo y el mundo que nos rodea; como consecuencia de ello, se ha creado un sinfín de oportunidades de comodidad, confort y eficiencia para el usuario final.

Ejemplos de éste aspecto creciente en cuanto al uso de NFC es el rápido aumento del contenido electrónico y la conectividad en los automóviles, las crecientes demandas de la creación de redes de banda ancha, electrónica industrial interconectada y la medicina electrónica, hogares conectados y la proliferación de dispositivos móviles inteligentes están todos impulsando la innovación tecnológica y seguridad que obligan a desarrollar soluciones inteligentes interconectadas.

Se considera a NFC como una plataforma abierta diseñada desde el inicio para teléfonos y dispositivos móviles. Debido a sus características de funcionamiento la aplicabilidad de NFC más que para la transmisión de grandes cantidades de datos es para comunicación instantánea, es decir, identificación y validación de equipos/personas. (Nfc, 2016). Entre otras de las características sobresalientes sobresalen la velocidad de comunicación, que es casi instantánea sin necesidad de emparejamiento previo, adicionalmente, el alcance de la tecnología NFC es muy reducido, pues se 
mueve como máximo en un rango de los $20 \mathrm{~cm}$. A su favor también juega que su uso es transparente a los usuarios y que los equipos con tecnología NFC son capaces de enviar y recibir información al mismo tiempo. (NXP, 2016)

Casos Referenciales: La ciudad de Wuhan, en China tiene un proyecto piloto, donde se utilizarán pulseras para utilizar la tecnología NFC, así es como a estas pulseras se cargarán con una aplicación de tránsito, lo que les permitirá a los usuarios hacer uso de los autobuses y trenes metropolitanos con solo un movimiento de las manos. También incluirá un rastreador de actividad física, que les proporcionará la facilidad a los usuarios llevar un registro de sus actividades diarias, como cuántos pasos caminaron, la distancia que recorrieron y el patrón de sueño. Los usuarios pueden consultar el saldo y recargar la cuenta a través de una aplicación para teléfonos inteligentes. Wuhan es uno de los principales centros de transporte de China. Para asegurar el flujo armónico de pasajeros, el sistema de tránsito está implementando rápidamente la tecnología sin contacto. En este momento, hay más de 13 millones de tarjetas en uso. (Gelmato, Pulseras NFC, 2016)

Caso 1: Solución Optelio Contactless MiniTag: Es una nueva forma de pagar sin usar una tarjeta de plástico. La versátil solución presentada por ésta empresa, permite mejorar y ampliar su oferta sin contacto, ya que apunta a nuevos segmentos de clientes, tales como los jóvenes y los aficionados al deporte, así como a capitalizar las oportunidades de negocio en sectores emergentes, entre ellos, los estadios y los eventos corporativos. Está desarrollado para cualquier circunstancia: La simplicidad, la seguridad y la flexibilidad están en el corazón de Optelio Contactless MiniTag. Esta solución compatible con el estándar EMV se puede usar para lo siguiente: Pagos (MasterCard y Visa), Control de acceso, Transporte público, Redes sociales. (NXP, 2016)

Caso 2: Adicionalmente, existe un prototipo en Madrid que se inició en Febrero del 2015, para lo cual, el Consorcio Regional de Transportes ha firmado un convenio no sólo con la Empresa Municipal de Transportes (EMT), sino también con Telefónica, Gemalto SP y Samsung. Los móviles que se utilizan en la prueba piloto son de dicha marca y del modelo $\$ 4$, según han informado fuentes oficiales. Por eso, a través del móvil o de la Tablet, el usuario puede moverse por dentro de los intercambiadores y proyectar su viaje con más facilidad. Además, se han desarrollado aplicaciones para que cada usuario, con un Smartphone una Tablet pueda consultar toda la información referente a su tarjeta de transporte público.

\section{Descripción de la solución en Madrid:}

Considerando la complejidad tecnológica del proyecto, ha sido necesario contar con diversos actores (fabricante de teléfonos, operador de comunicaciones, medio de pago o entidad bancaria, fabricante de tarjeta SIM, integrador de tecnología, etc.). Como consecuencia, la solución técnica adoptada en el proyecto ha sido posible gracias a la participación de las siguientes empresas, cada una de las cuales ha jugado un papel fundamental y necesario en el mismo: 
- Consorcio Regional de Transportes Madrid (CRTM): autoridad de transporte que concentra las competencias en materia de transporte regular de viajeros en la Comunidad de Madrid. Es el propietario del sistema TTP, Tarjeta de Transporte Público sin contacto, definido para el transporte en Madrid.

- Empresa Municipal de Transportes de Madrid (EMT): sociedad anónima, propiedad del Ayuntamiento de Madrid, encargada de la prestación del servicio de transporte público urbano colectivo de superficie mediante autobús en la ciudad de Madrid. La EMT ha realizado los desarrollos técnicos del BackOffice necesarios en la generación de los comandos DESFire, que permiten la personalización y recarga de los títulos de transporte de forma remota a través del Trusted Service Manager (TSM).

- Gemalto: compañía privada de seguridad digital, responsable de proveer la infraestructura del Trusted Service Manager (TSM) necesaria para la escritura remota en las SIMs de los usuarios de los títulos de transporte y de la ejecución de los comandos generados por la EMT. Asimismo, las SIMs NFC compatibles con el servicio han sido fabricadas por Gemalto.

- Telefónica: multinacional española de telecomunicaciones que ha proporcionado la red móvil y los servicios móviles imprescindibles para la prestación final del servicio en las SIMs de los usuarios.

- Samsung: fabricante multinacional de dispositivos electrónicos que ha proporcionado los desarrollos software imprescindibles para la generación de la aplicación que sirve a los usuarios para la gestión de los títulos de transporte instalados en la SIM, así como los terminales móviles NFC. A su vez, sirve de interfaz para la recarga de dichos títulos.

- BBVA: entidad bancaria que ha proporcionado la pasarela de pagos online a través de la cual se realiza la compra y recarga de los títulos de transporte.

El flujo normal (simplificado) de una compra por parte de un usuario sería según se describe a continuación:

1. Una vez el usuario dispone de una tarjeta SIM personalizada y a través de la aplicación móvil desarrollada al efecto, el usuario inicia la recarga del título. Para ello se consulta al BackOffice los títulos y precios disponibles.

2. Una vez seleccionado el título a recargar, el usuario inicia la compra a través de la pasarela de pagos.

3. Si la compra ha tenido éxito, se notifica dicho éxito al BackOffice, quien generará los comandos necesarios para dicha recarga. Todo ello se envía a la SIM, de forma segura, a través del TSM.

4. EI TSM, a través de la red móvil, hace llegar el título de transporte a la SIM, donde se almacena de forma segura. 


\section{Aplicación de gestión}

Se ha desarrollado una aplicación Android para el dispositivo NFC que provea de la funcionalidad necesaria para la gestión de los títulos de transporte. Para su utilización, la aplicación permitirá un doble uso:

1. Interacción con el usuario: la aplicación provee de acciones específicas que el usuario podrá realizar para obtener información y ejecutar operaciones sobre su título de transportes. En concreto, se podrá consultar el saldo de viajes disponible y solicitar una carga.

2. Durante el proceso de validación en un medio de acceso de transporte, la aplicación detectará el cambio de saldo y devolverá una información de la validación producida y el saldo resultante.

La aplicación Android desarrollada es el complemento perfecto para mejorar la usabilidad del título de transporte buscando la sencillez y la reducción de los menús al máximo. Esto a su vez mejora la experiencia del usuario. De esta manera, al iniciar la aplicación y de una forma automática, el usuario puede observar el saldo pendiente de viajes. Por último, en caso de querer recargar el título de viaje, podrá hacerlo pulsando sobre el "carro de la compra" disponible en la pantalla principal. Este icono proporciona acceso al entorno de la pasarela de pagos, donde poder realizar la compra del título contra una tarjeta de crédito. (Facility, 2016).

Principios de Funcionamiento: Se trata de un sistema de transmisión de datos similar al bluetooth y que utiliza los principios de la tecnología RFID (identificador por radiofrecuencia). Además, ofrece prestaciones mucho más amplias que la RFID porque aprovecha el extendido uso de los teléfonos móviles y sus capacidades de cómputo. En la Figura 2 podemos observar el funcionamiento y una variedad de aplicaciones sobre NFC.

\section{ESPECIFICACIÓN DE LA ARQUITECTURA NFC}

A través de la utilización de los elementos clave en las normas existentes y reconocidas como ISO / IEC 18092 e ISO / IEC 14443-2,3,4, así como JIS X6319-4, las especificaciones NFC Forum establecen un estándar tecnológico que armoniza las normas y se extiende sin contacto existentes el desbloqueo de todas las capacidades de la tecnología NFC en los diferentes modos de funcionamiento sin contacto, el modo de igual a igual, lector / escritor modo, el modo de emulación de la tarjeta. (Forum, 2017)

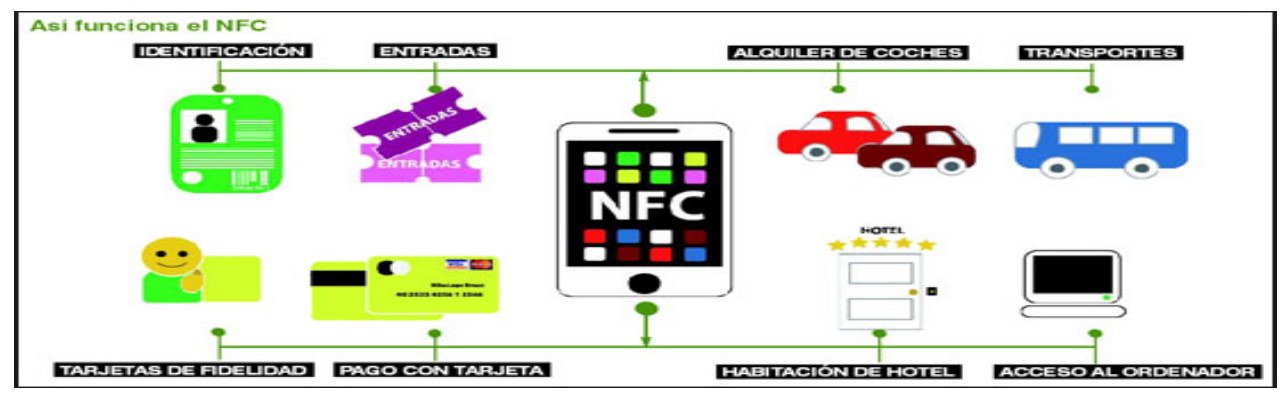

Figura 2. Aplicaciones de NFC (Evolucion, 2016)

Enfoque UTE, V.7-Sup.1, Feb.2017, pp.31- 45 
"Se considera a NFC además como un enlace crítico al Internet de las cosas: Los usuarios que acceden a Internet de forma inalámbrica a través de dispositivos móviles pronto serán la mayoría. Entre las diversas opciones de apareamiento móvil, sólo NFC está específicamente diseñado y desarrollado para maximizar la privacidad a la vez que tiene una configuración de poder cero, y realizando ambos de una manera muy rentable" (XatakaAndroid, 2016)

A continuación la Figura 3 en la cual se detalla la Arquitectura NFC.

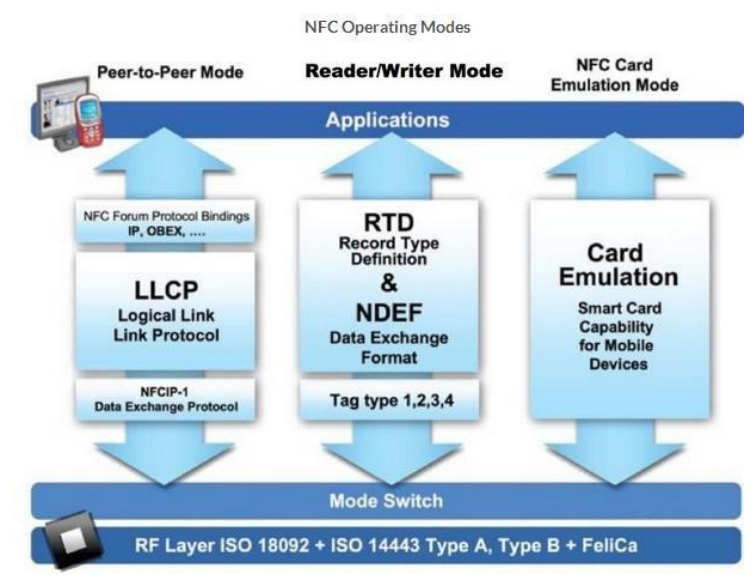

Figura 3. Estructura de NFC (DigiKey, 2016)

Entre las características que se destacan están:

A. Privacidad: NFC por diseño tiene un limitado campo de operación, lo que impide la escucha de datos que podría ocurrir desde una distancia mayor. También requiere controlar la intención de la aplicación de un dispositivo habilitado con NFC a un objeto dotado de la tecnología NFC para leer su memoria. Este enfoque está en contraste con los protocolos tales como $\mathrm{Wi}-\mathrm{Fi}$, que requieren radios para transmitir información sin importar la intención.

B. Configuración de poder cero: Al establecer comunicación entre un lector NFC y un transpondedor NFC (etiqueta), la energía obtenida a partir del campo de RF de la energía acumulada en el lector de la etiqueta, proporciona conectividad a los dispositivos de Internet de las Cosas (IOT) sin necesidad de utilizar baterías o corriente lo cual permite una configuración con ausencia de éstos elementos. Esta característica de recolección de energía permite un número de aplicaciones de baja potencia y de bajo costo.

C. Soluciones rentables: La adición de una etiqueta NFC conectada a un sistema embebido puede establecer la conectividad a dispositivos móviles a un costo mucho más bajo que los enfoques Bluetooth o WiFi. Además, eliminando la necesidad de una batería en un sistema embebido el requerimiento de materiales usado es menor y consecuentemente su costo baja. 
D. NFC y el transporte: El valor de uso de tarjetas inteligentes sin contacto en los sistemas de tarifas de transporte masivo ofrece comodidad al pasajero y al operador tales como: la velocidad de embarque, tiempos de espera reducidos, más información de gestión, nuevas opciones para los programas de los empleadores locales y una mayor flexibilidad de la política de tarifas. Como resultado, las agencias de transporte de todo el mundo han puesto en marcha los sistemas de pago de tarifas de tarjetas inteligentes sin contacto, de valor almacenado normalmente o de paso almacenado Implementaciones en las que la tarjeta posee un producto de tarifa o valor en efectivo que se actualiza con cada uso de la tarjeta. Con el advenimiento de los sistemas de pago sin contacto abiertos de las marcas de pago y los emisores de tarjetas bancarias, las agencias de transporte ven una oportunidad para mejorar el servicio al cliente y la eficiencia operativa mediante la reducción del papel como emisor de medios de pago y adquirente de transacciones Por lo tanto es conveniente el análisis de las oportunidades y los retos de la utilización de crédito sin contacto emitida por el banco, tarjetas de débito y tarjetas de prepago para el pago de tarifas de tránsito y de cómo las dos industrias pueden vincular los productos y servicios de pago. (Alliance, 2016)

En los últimos años, se ha introducido el pago sin contacto, por lo cual la industria de tarjetas bancarias ha generado programas que hacen las tarjetas tradicionales más atractivas para las transacciones de bajo valor. Reconociendo que las transacciones por debajo de \$ 25 se llevan a cabo en entornos que requieren transacciones más rápidas, las marcas de pago han adoptado programas que faciliten las transacciones mediante la eliminación de los requisitos de procesamiento que requieren mucho tiempo, como las firmas y recibos. Las transacciones sin contacto no requieren conexión física entre el dispositivo del consumidor el pago (normalmente una tarjeta) y el terminal del comerciante de punto de venta (POS). El pago sin contacto es simplemente una forma diferente de presentar una tarjeta con el sistema POS, para el consumidor, hay poca diferencia entre la funcionalidad de una tarjeta de crédito sin contacto y una tarjeta estándar del banco de banda magnética. La transacción también incluye un contacto, criptograma dinámico único, que identifica cada operación exclusivamente para evitar transacciones fraudulentas.

\section{Metodología}

Se realiza una investigación exploratoria para lo cual, se toma como base un Caso de Estudio acerca del Metro de Quito para posteriormente realizar una propuesta para la solución de pagos. Veamos el caso del Metro de Quito: su propósito es integrar el Sur y el Norte de la Ciudad, entre Quitumbe y El Labrador en apenas 34 minutos, con 8 trenes de seis vagones cada uno que transportarán hasta 400 mil personas en un día. En su trayecto, tendrá 15 estaciones de cómoda 
accesibilidad para personas de capacidades reducidas. Desde la interpretación cartográfica y geológica hasta el monitoreo sísmico y neotectónico, pasando por lo arqueológico y patrimonial, conforman la estructura de los once estudios de soporte sobre los que Metro de Madrid, realizó el diseño e ingeniería definitivos de la Primera Línea del Metro de Quito, partiendo de las características únicas de una ciudad Patrimonio de la Humanidad, con fuentes de agua subterránea, quebradas, rellenos y suelos de tipología diversa, que determinaron la necesidad de investigar, conocer e interpretar con mayor precisión las características propias de Quito, a través de estudios técnicos y científicos que se iniciaron con la interpretación cartográfica de la Ciudad

A. Requerimientos NFC para transporte:

Los Sistemas de tránsito de la AMT (Agencia Metropolitana de tránsito) manejan una serie de funciones, incluyendo la emisión de la tarjeta de pago, la aplicación de normas específicas de la agencia para determinar la tarifa que se cobra cuando se utiliza la tarjeta de pago, y procesar el pago. Para cumplir estas funciones, los sistemas de tránsito de la AMT deben cumplir estos requisitos cruciales.

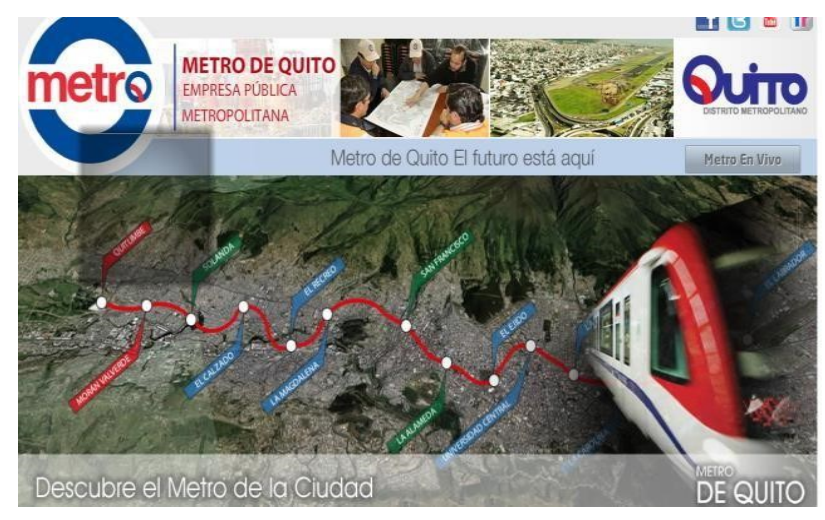

Figura 4: Proyecto Metro Quito ${ }^{3}$

B. Arquitectura de Control y Registro:

Un enfoque de tarjetas bancarias sin contacto abierto para el pago de la tarifa de tránsito utiliza una arquitectura basada en cuentas, en lugar de la arquitectura basada en una tarjeta tradicional. En un sistema que utiliza una arquitectura basada en cuentas, el terminal lee la información almacenada en una tarjeta inteligente sin contacto y lo envía de vuelta a un back office través de una red de comunicaciones. El sistema de back office (o host) mantiene la lógica del sistema y determina si la tarjeta es válida y devuelve una señal que permite al terminal abrir la puerta o señalar al piloto y al operador de autobuses si se debe permitir el paso.

El terminal puede realizar funciones de seguridad, y también puede comprobar una lista negativa o positiva para determinar validez de la tarjeta antes de enviar los datos de pago para el huésped.

3

http://cidoc.es/blog/proyecto-ejecucion-de-la-primera-linea-del-metro-de-quito-fase-2/ 
El sistema de back office utiliza los datos enviados por el terminal para aplicar las reglas de negocio relevantes y determinar un precio para la transacción utilizando la política de tarifas / reglas de la agencia. Los tipos de transacciones y formas de pago pueden variar con esta arquitectura basada en reglas de negocio de la agencia de tránsito y de los tipos de tecnologías que se utilizan.

\section{Discusión:}

En ésta sección, se realiza un análisis de las mejores alternativas a fin de encontrar una solución adecuada para los medios de pago electrónicos. Se ha realizado una verificación de varias propuestas, ya que existe un gran crecimiento en la utilización de NFC y las aplicaciones de la industria móvil, se han encontrado algunas opciones. Como fase complementaria se hace el análisis de Cnx Soft - Embeded Development (Soft, 2016), una empresa que trabaja en el campo de móviles con mucho éxito y diversas implementaciones y que es la que más se ajusta dentro del perfil requerido. Se encuentra que el sistema básicamente deberá constar de tres módulos: módulo de Creación de Tickets, Modulo de Validación de Tickets y Modulo de Venta de Tickets, mas no se especifica plataforma alguna para implementarlo. a)EI Modulo de Creación de Tickets: Se asume que está destinado a establecer el ingreso inicial y registro de usuario, así como activar los mecanismos de disparo de la aplicación, como tarjetas NFC, creación de usuarios y contraseñas. b) El Modulo de Validación de Tickets: Se asume que está destinado a validar los usuarios; así como el saldo a vigencia tarjetas NFC. c) El Modulo de Venta de Tickets: Se asume que está destinado a conectar el procesamiento transaccional de pago de los usuarios; así como activación o negación de servicio. Es pertinente además mencionar que, aunque la tecnología NFC ha tenido una gran difusión en los últimos años, con la llegada de los dispositivos iPhone 6, iPhone 6 Plus y Apple Watch por fin tenemos un chip NFC con el que los usuarios podrán hacer transacciones sin contacto gracias a Apple Pay. Sin embargo, esta funcionalidad no podrá utilizarse con terceras aplicaciones, ni con otro hardware. Apple no va a poner a disposición de los desarrolladores el chip NFC que tienen los nuevos dispositivos, al menos, durante el primer año, deberemos entonces esperar que para las nuevas versiones existan acuerdos para lograr alternativas en éste sentido.

La Figura 5 muestra la propuesta inicial, que posteriormente será considerada para desarrollar una nueva alternativa en el sistema de pagos utilizando NFC, 


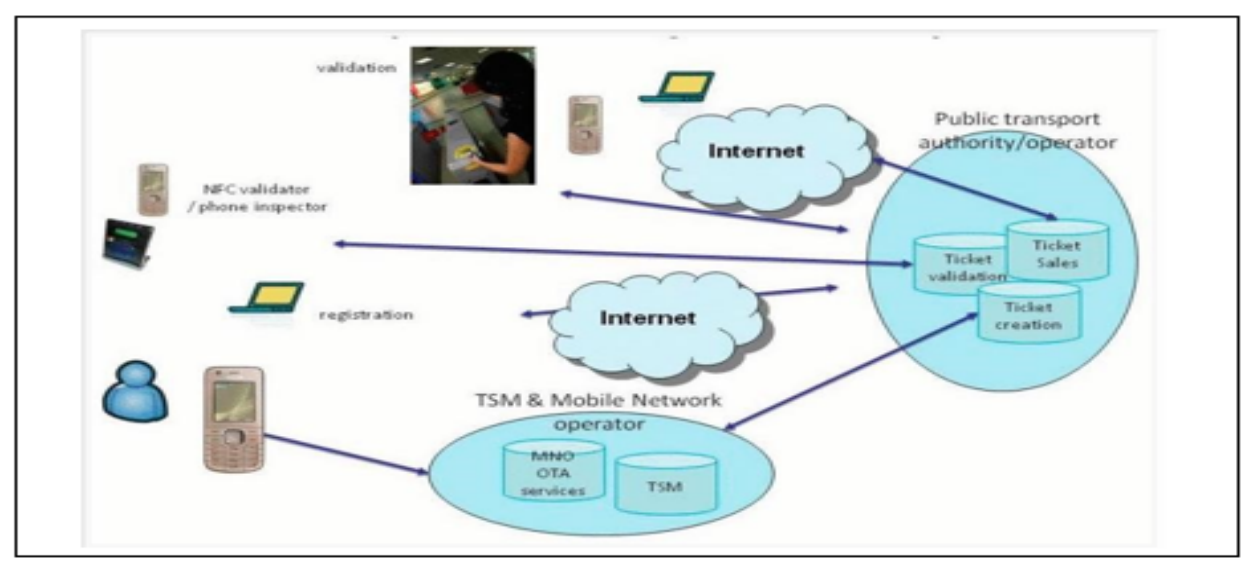

Figura 5: Propuesta Inicial ${ }^{4}$

A. Ventajas de la propuesta

i) Un socio de confianza para el despliegue de servicios NFC y aprovisionamiento seguro de administración de credenciales lo cual se refiere a Gestión de Servicios de confianza, de acuerdo a (Gelmato, Gelmato, 2016). "Gestión de Servicios de confianza es una parte neutral que actúa como un agregador de servicio y orquestador en el despliegue de los servicios móviles, para vincular los proyectos entre los emisores (por ejemplo, los operadores de redes móviles, bancos, OTT ), los proveedores de servicios (al por menor, las líneas aéreas, los programas de promoción / fidelización, transporte) y terceros técnicos (proveedores de cartera, sistemas de venta de entradas ,etc.)"

ii) Se encuentra que el sistema en su globalidad estará operado por una empresa de transportes quien será la encargada de la administración del sistema.

iii) No se describen en detalle los elementos a ser utilizados.

iv) Incluye OTA (Over-The-Air) es una tecnología utilizada para comunicarse, descargar aplicaciones y gestionar una tarjeta SIM sin estar conectado físicamente a la tarjeta

B. Desventajas de la propuesta:

i) La arquitectura de hardware y software para aplicaciones de transporte requiere de un sistema de validación del usuario, bien sea que el usuario se conecte al sistema a través de la red cableada o inalámbrica, o directamente a un terminal del operador del sistema de transportes.

Se encuentra que en los puntos de acceso y registro debería existir un nivel de seguridad en redes como la inalámbrica, así como en la intranet de la empresa.

4 http://www.cnx-software.com/2011/01/31/near-field-communication-nfc-inpublic-transport/ 
ii) Si bien NFC tiende a convertirse paulatinamente en un estándar, hay proveedores y fabricantes como Apple, que se enfocan en el desarrollo de sus propias aplicaciones como Passbook (AppleSsfera, 2016)

\section{Resultados}

Luego de realizar el análisis de la propuesta inicial; se ha determinado que tiene varias desventajas como son la seguridad transaccional, que es un aspecto crítico, tratándose de pagos en línea, adicionalmente no existe una normalización de los procedimientos para que el usuario utilice la plataforma, por lo cual pueden existir errores al ser éste un sistema basado en cloud, es decir el punto importante es que se debe configurar un sistema que permita un nivel de escalabilidad y flexibilidad de crecimiento, además seguro con un control total de la transaccionalidad. Es importante además que se defina adecuadamente y detalladamente el requerimiento de usuario para poder crear un software que brinde las facilidades necesarias de operación al usuario.

\subsection{DESARROLLO DE UNA NUEVA PROPUESTA:}

Con el propósito de confrontar las desventajas iniciales de la propuesta, se desarrolla una nueva propuesta por lo cual se sugiere una nueva opción que pretende cubrir algunos aspectos como:1) Brindar seguridad al usuario a través el protocolo SSL, para encapsular las transacciones mediante;2) Normalizar los procedimientos de conexión del usuario, al dispositivo NFC y desde éste a la nube y viceversa;3) Establecer el requerimiento de software para el usuario, así como una o varias aplicaciones de escritorio que interactúan con él y con el proveedor de servicios. 4) Buscar un sistema en el Cloud que permita escalabilidad y seguridad y cuyo costo de servicios como SaaS, PaaS e laaS sean escalables y versátiles en su forma de gestión.

\subsubsection{Resultados de la Nueva propuesta:}

En base al análisis realizado y una vez detectada las virtudes y falencias de la propuesta analizada, se propone una nueva alternativa que pretende cubrir algunos aspectos como:1) Dotar de seguridad al usuario a través de encapsular las transacciones mediante el protocolo SSL; 2) Establecer claridad en los procedimientos de conexión del usuario, al dispositivo de conexión NFC y desde éste a la nube y viceversa; 3) Definir claramente la necesidad de desarrollo de la aplicación para el usuario, así como una o varias aplicaciones de escritorio que interactúan con el usuario y con el proveedor de servicios. A continuación, se puede observar en la Figura 6, el esquema de la nueva propuesta. 


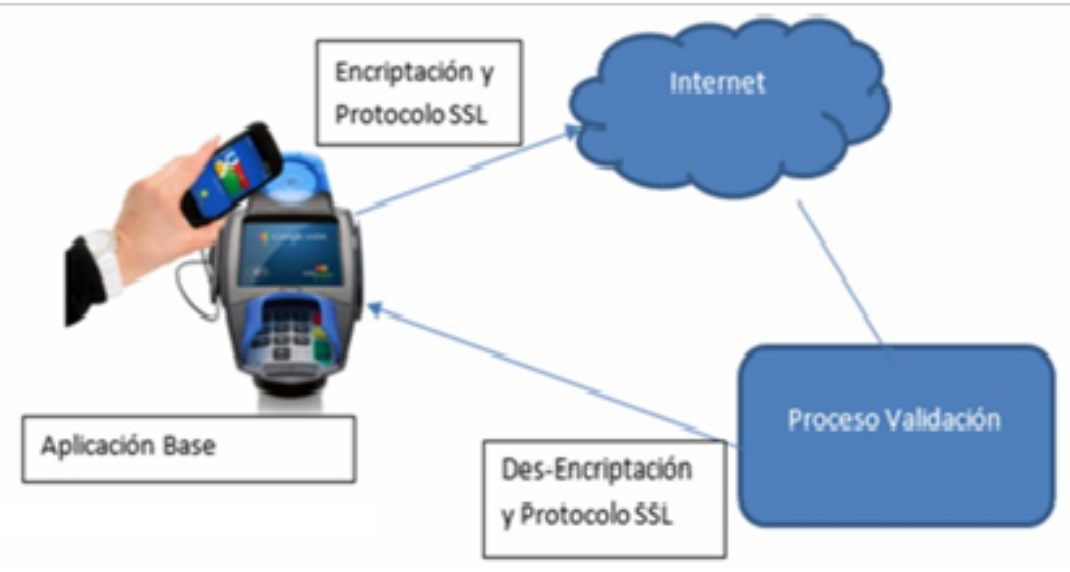

Figura 6. Descripción de la nueva propuesta

\subsubsection{Consideraciones en el Desarrollo de Aplicaciones}

A fin de lograr un alto nivel de seguridades se propone utilizar el protocolo de seguridad SSL.

- $\quad$ Características de SSL: SSL es un protocolo estratificado, por lo que en cada capa se le agregan a los mensajes campos con distintos tipos de contenido, como el largo de los mismos, algún tipo de descripción y la información propiamente dicha

- $\quad$ Framework de Desarrollo: Para el desarrollo de las aplicaciones se propone Eclipse a fin de garantizar compatibilidad con varios dispositivos vigentes en el mercado

- Aplicaciones a Desarrollarse: El teléfono deberá tener una aplicación, la misma que al acercar al lector de NFC disparará la aplicación de verificación y permitirá el acceso o no del usuario, entonces se enviara la información almacenada en la etiqueta del teléfono.

- Aplicación Móvil: Adicionalmente se debe considerar que se requiere el desarrollo de aplicaciones para poder recibir y enviar información, así como de una aplicación web para poder realizar la compra de recargas de tiempo, tickets, etc.

- $\quad$ Aplicación de Escritorio: Se requiere de una aplicación de escritorio para poder autorizar transacciones, recibir y enviar información, así como de una aplicación web para poder realizar la compra de recargas de tiempo, tickets, etc. El desempeño de los sistemas se basa en tres pasos: 1) El sistema es una combinación de la plataforma de comunicación basada en la nube y el entorno de ejecución de aplicaciones 2) La aplicación y transmisión de datos se realiza de forma automática desde la nube 3) Las solicitudes se activan y pueden correr en el dispositivo (PC, portátil o Smartphone) y se eliminan cuando no se requieren más. La tecnología NFC permite a los dispositivos conectarse entre sí, tanto los datos y las aplicaciones requeridas pueden ser transferidos de un dispositivo a otro. 


\subsubsection{Descripción de la aplicación:}

A continuación se muestra la descripción del proceso de ejecución de la transacción:

1) El terminal POS para hacer un pago, 2) La aplicación de pago se descarga desde la nube en el teléfono móvil de almacenamiento temporal seguro del cliente, 3)Terminal de punto de venta se comunica con el proveedor de la nube para comprobar si el cliente tiene suficiente crédito, 4) Proveedor de nube transfiere la información requerida para POS, 5) Sobre la base de la información que se envió al comerciante, terminal punto de venta o bien autoriza o rechaza la solicitud del cliente de transacción, 6) POS: Se comunica con la nube para actualizar la cuenta del cliente - si la petición del cliente está autorizada, el importe de la compra es retirado de la cuenta del cliente, de lo contrario no habría la modificación a su cuenta.

\section{Conclusiones}

- Lo importante en el uso de tecnologías NFC es facilitar al usuario el acceso a transacciones mediante el aprovechamiento de las potencialidades que la nueva tecnología ofrece y el mercado lo demanda.

- La tecnología NFC ofrece ventajas frente a los códigos QR en los casos en que se desea aprovechar el elemento de la proximidad o se requiere ejecutar una instrucción para agilizar un proceso.

- Gracias a los dispositivos móviles y la conexión a internet cada vez tenemos más medios a nuestro alcance para facilitar a los usuarios el acceso a la información. Es el momento de encontrar la forma de explotar esos medios y buscar soluciones óptimas, al menor coste posible, que realmente funcionen y sean útiles y eficaces para nuestros usuarios.

- El popular uso de la tecnología NFC está en crecimiento lo que hace avizorar que los medios de pago y el loT, se beneficiarán de un sistema muy innovador y práctico, beneficiando a los proveedores de redes móviles, a los proveedores de servicios, y a los usuarios.

- Otra de las alternativas es utilizar Información Basada en Localización "LBS" (Location Based Systems), que ayudaría al usuario a ubicarse fácilmente entre paradas a fin de evitar demoras en las transferencias entre líneas, muy útil para personas de la tercera edad y con discapacidad visual. 
- Deberemos esperar además que se den acuerdos adicionales entre Apple y los fabricantes y proveedores de NFC para lograr beneficios para los usuarios de IPhone.

\section{Bibliografía}

Al, D. e. (2011). Big Data and Cloud Computing: Current State and Future Opportunities. roceedings of the 14th International Conference on Extending Database Technology (págs. 530-533 ). New York, NY, USA: EDBT/ICDT '11. doi:10.1145/1951365.1951432

Alliance, S. (08 de 20 de 2016). SMART CARD ALLIANCE. Recuperado el 08 de 20 de 2016, de SMART CARD: http://www.smartcardalliance.org/.

AppleSsfera. (12 de 10 de 2016). AppleSsfera. Recuperado el 12 de 10 de 2016, de https://www.applesfera.com/aplicaciones-ios-1/passbook-una-aplicacion-que-puedeofrecer-mucho-en-un-futuro-cercano

Cidoc. (30 de 10 de 2016). Cidoc. Recuperado el 01 de 10 de 2016, de http://cidoc.es/blog/proyectoejecucion-de-la-primera-linea-del-metro-de-quito-fase-2/

DigiKey. (01 de 11 de 2016). DigiKey. Obtenido de http://www.digikey.be/en/articles/techzone/2013/feb/lower-cost-integrated-solutions-enablenew-nfc-wireless-apps

Evolucion. (10 de 11 de 2016). Evolucion. Recuperado el 10 de 11 de 2016, de evolucion: http://www.e-volucion.es/2012/06/tecnologia-nfc-entra-Ileno-nuestro-movil

Facility. (26 de 11 de 2016). Facility Management and Services. Recuperado el 26 de 11 de 2016, de Facility Management and Services: http://www.facilitymanagementservices.es/tendencias/smart-cities/sistema-de-acceso-nfcpara-el-transporte-publico-de-madrid

Forum, N. (01 de 01 de 2017). NFC Forum. Obtenido de http://nfc-forum.org/our-work/specificationsand-application-documents/specifications/

Gelmato. (01 de 12 de 2016). Gelmato. Obtenido de http://www.gemalto.com/latam/serviciosfinancieros $/ \mathrm{mfs} / \mathrm{tsm}$

Gelmato. (2 de 6 de 2016). Pulseras NFC. (http://www.gemalto.com/press/Pages/Las-pulserasNFC-de-Gemalto-agilizan-el-transporte-en-China.aspx) Recuperado el 08 de 08 de 2016, de 
Brochure NFC: http://www.gemalto.com/brochures-site/download-site/Documents/fscontactless-payment-esp.pdf

Nfc, S. (25 de 12 de 2016). Shop Nfc. Recuperado el 28 de 12 de 2016, de Shop Nfc: http://www.shopnfc.it/es/content/6-caracteristicas-tecnicas-de-etiquetas-nfc

NXP. (25 de 12 de 2016). nfc-forum. (NXP) Recuperado el 28 de 12 de 2016, de SlideShare: http://www.slideshare.net/NFC-Forum/nfc-everywhere

Shannon, C. (s.f.).

Soft, C. $(10$ de 08 de 2016). CNX-SOFT. Obtenido de http://www.cnxsoftware.com/2010/12/28/near-field-communication-nfc/

University, B. D. (15 de 10 de 2016). Big Data University. Obtenido de Big Dta University Course: https://courses.bigdatauniversity.com/courses/coursev1:BigDataUniversity+BD0101EN+2016/courseware/407a9f86565c44189740699636b4fb8 5/5954a0d4016a4346b83ff34bd5edef84/

XatakaAndroid. (01 de 08 de 2016). XatakaAndroid. Obtenido de https://www.xatakandroid.com/aplicaciones-android/momo-pocket-el-pago-con-el-movilnunca-fue-tan-sencillo 